\title{
Gangliosides in lesion-induced synaptogenesis: studies in the hippocampus of the rat brain
}

\author{
Daniel Masco and Wilfried Seifert \\ Department of Neurobiology, Max-Planck Institut für Biophysikalische Chemie, Goettingen (F.R.G.)
}

(Accepted 12 September 1989)

Key words: Ganglioside; Lesion; Brain injury; Sprouting; Hippocampus; Area dentata

\begin{abstract}
Changes in ganglioside composition, biosynthesis and individual distribution were studied in hippocampal regions after unilateral destruction of the entorhinal cortex. After 1 and 3 days postlesion (dpl), a decrease in ganglioside content was detected in area dentata (AD) and pyramidal cell regions CA1-CA3 (CA), both ipsilateral and contralateral to the lesion. By $5 \mathrm{dpl}$ all the values had returned to control values, except in $\mathrm{AD}$ which showed a dramatic increase in total ganglioside content reaching a maximum at $12 \mathrm{dpl}$. By $30 \mathrm{dpl}$ this area also showed control content. A significant increase in biosynthesis of gangliosides was observed at 5 and $8 \mathrm{dpl}$ in the hippocampus ipsilateral to the lesion without changes in the contralateral counterpart. Individual ganglioside distribution showed a pronounced change in GM1 and GQ1b with small changes in the other major gangliosides. Significant differences were observed in the distribution of gangliosides between the two hippocampal regions studied in unoperated control animals. GD1a was more concentrated in AD, whereas GQ1b, GT1b and GD1b predominated in CA. The data presented here indicate that important modifications in ganglioside content as well as pattern occur in the deafferented hippocampus, a phenomenon that could be related with the known effect of gangliosides on neuritogenesis observed in cell culture studies.
\end{abstract}

\section{INTRODUCTION}

Ablation of the entorhinal cortex results in a deafferentation of the hippocampus ipsilateral to the lesion. Over a period of about two weeks, the axons remaining within the hippocampus begin to branch and replace the degenerating axons in a process called reactive sprouting. While an almost complete description of the cellular events during this remodeling and plasticity is becoming available $^{5}$, little work has been done on the molecular mechanisms that underlie these effects. Among the molecules that might play a role during this process are gangliosides. These sialo-glycosphingolipids, which are among the most abundant lipids in the central nervous system $^{21}$, are localized in the outer leaf of the lipid bilayer of the plasma membrane exposing to the external environment their negatively charged polysaccharide chains ${ }^{60}$.

One of the most intriguing facets of gangliosides is the pronounced changes they undergo, both qualitatively and quantitatively, during the course of neuronal development, growth and differentiation in vivo $14,19,20,30,44$, $45,53,54,56,63$ as well as in neuronal cell culture ${ }^{6,8,9,37,61,62}$. The functional involvement of gangliosides in development was reinforced by the discovery in our laboratory that exogenously administered gangliosides increase survival and induce differentiation in the neuroblastoma cell line $\mathrm{B} 104^{31}$. Many other workers have corroborated this participation of gangliosides in differentiation processes using a variety of cultured cells $\mathbf{s}^{3,7,10,22,27,42,51}$.

Further evidence that also points to a possible role of gangliosides during development comes from studies of GM1 gangliosidosis. In this disease an excessive accumulation of gangliosides leads to the formation of meganeurites which show dendrite-like processes, growth cones, and spines ${ }^{39}$. Thus an abnormal increase in endogenous gangliosides produces a neuronal growth phenomenon.

One of the most common approaches to study ganglioside function is administration of exogenous gangliosides in vivo $^{28}$. Using this strategy, gangliosides were clearly demonstrated to promote repair processes after different kinds of lesions ${ }^{1,4,36}$. Such treatment has repeatedly been shown to improve behavioral performance after brain damage $\mathrm{e}^{11,17,38,40,41,46,47}$. These several lines of evidence support the proposal that gangliosides may play a functional role during reactive sprouting and remodeling plasticity.

A necessary first step in the investigation of the possible role of these molecules in compensatory axonal

Correspondence: W. Seifert, Department of Neurobiology, Max-Planck Institut für Biophysikalische Chemie, Am Fassberg, 3400 Goettingen, F.R.G. 
growth will be the characterization of the total content as well as distribution of individual gangliosides in a brain region during the period in which the axonal, synaptic and dendritic remodeling is occurring. In the present paper we investigated changes in ganglioside content and pattern occurring in the hippocampus following entorhinal lesion in an attempt to correlate these changes with specific morphogenetic events.

\section{MATERIALS AND METHODS}

\section{Surgery and tissue preparation}

Young adult albino male rats (Wistar strain, from a colony housed in our Institute) weighing $220-280 \mathrm{~g}$ were used in this study. Unilateral lesions were made under ether anesthesia in a stereotaxic apparatus. The skull overlaying the transverse sinus was removed. The electrode was inserted into the right side of the brain at 3 points (with a lateral distance of $1 \mathrm{~mm}$ between points) and $1 \mathrm{~mA}$ of current was applied for $45 \mathrm{~s}$ to a total of 9 different positions in order to eliminate both the lateral and the medial part of the entorhinal cortex $^{24}$. The animals were kept for 1 to 30 days after surgery, then decapitated and the brains were rapidly removed. At this time the lesioned areas were inspected visually to determine whether the lesions were sufficiently complete and comparable to each other. Two animals were used exclusively for histological analysis. In these animals horizontal Nissl-stained sections through the entorhinal cortex were analyzed (Fig. 1).

If the entorhinal lesion was judged to be adequate, the ipsilateral and contralateral hippocampus was isolated and cut into slices of 500 $\mu \mathrm{m}$ thickness on a McIlwain tissue chopper. Two hippocampal subregions were dissected from each slice under a binocular microscope: dentate gyrus (area dentata, $\mathrm{AD}$ ) and pyramidal areas CA1-CA3 (CA).

Survival times after entorhinal lesion were chosen taking into account the morphological studies done by others ${ }^{5,26,49,55}$.

\section{Lipid extraction}

Tissue samples (8-20 $\mathrm{mg}$ of protein) of the hippocampus of lesioned animals or unoperated controls were transferred to Eppendorf tubes and $360 \mu \mathrm{l}$ of distilled water was added. Samples were sonicated at room temperature for $30 \mathrm{~s}$ and aliquots for protein estimation were taken. Pilot experiments proved that sonication of these samples rendered the same tissue disruption as using glass homogenizer. Ganglioside extraction and purification were performed according to the procedure described by Svennerholm and Fredman ${ }^{59}$, which employs tissue extraction in chloroform:methanol:water $(4: 8: 3)$ followed by partitioning of the gangliosides into the aqueous phase. Gangliosides extracted by this method were clarified by centrifugation in order to remove particulate matter. Total sialic acid content of the samples was measured using a scaled-down variation of the method of Svennerholm ${ }^{57}$. Protein was determined by the Lowry method ${ }^{25}$. Protein content does not change in the whole dentate gyrus following entorhinal lesion ${ }^{32}$.

\section{Analysis of ganglioside pattern}

Aliquots of 5-10 $\mu \mathrm{g}$ ganglioside sialic acid were dried under nitrogen, resuspended in chloroform:methanol $(2: 1)$ and spotted as 5-7 $\mathrm{mm}$ bands on high performance thin layer chromatography plates (HPTLC) (Merck, Darmstadt, F.R.G.). A mixture of authentic gangliosides (Sigma, St. Louis, MO, U.S.A.) was spotted as reference. Plates were developed in chloroform:methanol:0.25\% aqueous calcium chloride $(55: 45: 10)$ in a chromatography tank at room temperature for $1 \mathrm{~h}$. After that the plates were air-dried, sprayed with resorcinol reagent ${ }^{57}$, covered with a glass plate and heated to $90{ }^{\circ} \mathrm{C}$ for $15 \mathrm{~min}$ to visualize individual bands. Ganglioside patterns were quantified by densitometry using a Shimadzu CS-930 dual wavelength TLC scanner. A technical consideration has to be mentioned. Using HPTLC as the method to study ganglioside patterns, we observed that the calculated relative concentration of gangliosides varied to a great deal when densitometric evaluation was done on different plates. The reason for this is probably the virtual impossibility to exactly reproduce the application to the HPTLC plate of the spraying reagent. To cope with this problem, the densitometric measurement of the resorcinol-positive compounds of all samples of a particular time after lesion, i.e. ipsilateral as well as contralateral samples of both, $A D$ and $C A$, was done on the same plate and compared with the unoperated control region also on the same plate. This procedure enabled us to estimate the relative distribution of all major gangliosides and calculate the changes with respect to control with great reproducibility. The Mann-Whitney $U$-test and sign test were used for statistic analysis.

The ganglioside nomenclature of Svennerholm ${ }^{58}$ is used throughout this paper.

\section{Injection of $I^{3} \mathrm{H} / \mathrm{glucosamine}$}

Animals which had survived entorhinal lesions for 1-30 days as well as unoperated controls were ether-anesthetized, placed in a stereotaxic frame and intraventricularly injected with $5 \mu \mathrm{l}(1 \mu \mathrm{l} / 2$ min) buffered saline containing $500 \mathrm{Ci}$ of $\mathrm{D}-\left[1,6^{-3} \mathrm{H}\right]$ glucosamine hydrochloride ( $42.5 \mathrm{Ci} / \mathrm{mmol}$, New England Nuclear, Boston, MA, U.S.A.) using a Hamilton syringe. The needle was withdrawn from the animal $10 \mathrm{~min}$ after the injection was complete. The animals were left to survive for $24 \mathrm{~h}$ and then decapitated. Ipsilateral as well as contralateral hippocampus and the cerebellum were immediately removed from the brains, frozen in liquid nitrogen and stored at $-80^{\circ} \mathrm{C}$ until further processing. The hippocampus was cut into slices as described above and sonicated in $500 \mu \mathrm{l}$ water, lyophilized to remove the radioactive water and resuspended in $360 \mu \mathrm{l}$ water and sonicated for $1 \mathrm{~min}$. The samples were dialyzed for $24 \mathrm{~h}$ using benzoylated dialysis tubing (Sigma, St. Louis, MO, U.S.A.) which has a molecular cut-off of approximately $2000 \mathrm{Da}$. After dialysis, gangliosides were isolated from these samples as described above.

Cerebellum (80-100 mg wet weight) was homogenized with $5 \mathrm{ml}$ of water in a glass homogenizer and lyophilized. Ganglioside

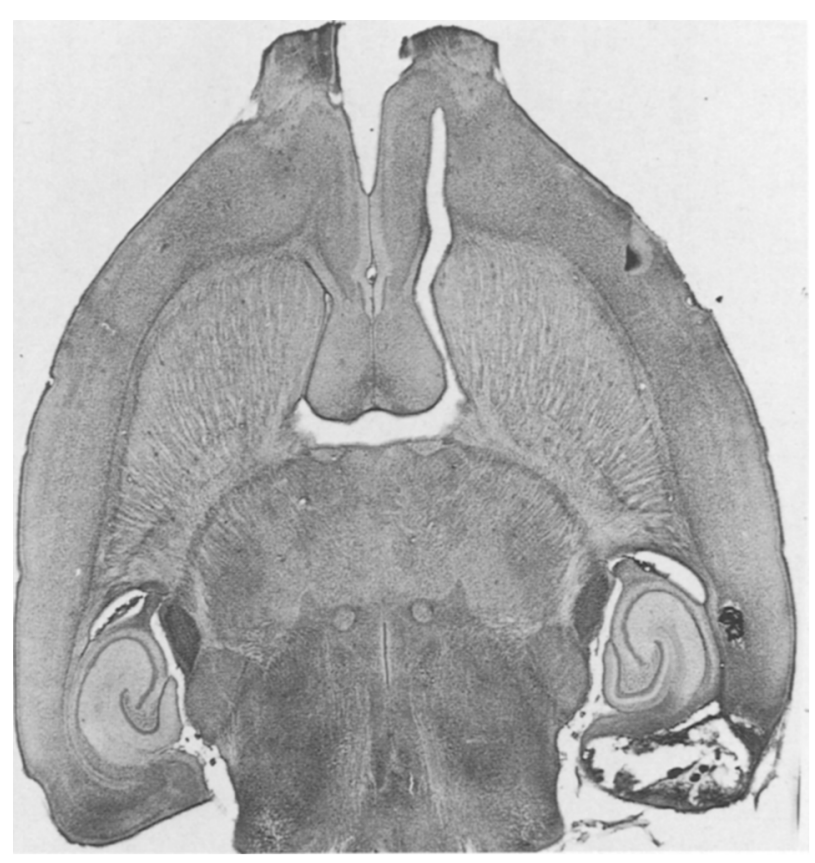

Fig. 1. Example of horizontal Nissl-stained section through the temporal part of the hippocampal region showing the extent of a typical entorhinal cortex lesion. The lesion usually included the parasubiculum and parts of the adjacent neocortex and presubiculum. 
extraction and purification was achieved as before but increasing the proportion of solvents takes the tissue amount into account.

Total gangliosides were separated by HPTLC, and the bands were visualized by iodine vapor in order to leave the ganglioside intact. Identification of ganglioside bands was achieved by comparison of their chromatographic mobilities with standards. All the gangliosides were then scraped off the plates and placed into scintillation vials. $1 \mathrm{ml}$ of water:methanol (1:1) was added and the vials were sonicated to solubilize the gangliosides. After adding $15 \mathrm{ml}$ of Aquasol (New England Nuclear, Boston, MA, U.S.A.) the vials were vortexed for $1 \mathrm{~min}$ and the radioactivity determined in a Packard $2000 \mathrm{CA}$ liquid scintillation counter. The counting condition was rigorously tested for being valid for samples containing silica gel.

In two plates radioactive spots were localized using ${ }^{3} \mathrm{H}$-Ultrofilm (LKB, Bromma, Sweden) to visualize all the radioactive products obtained in the ganglioside fraction.

\section{RESULTS}

\section{Direct analysis of gangliosides in lesioned animals}

The lesions of the present study included all of the medial and lateral subdivisions of the entorhinal cortex, part of the parasubiculum and the presubiculum (Fig. 1). In no case was damage to $\mathrm{AD}$ observed. This lesion resulted in virtually complete removal of entorhinal

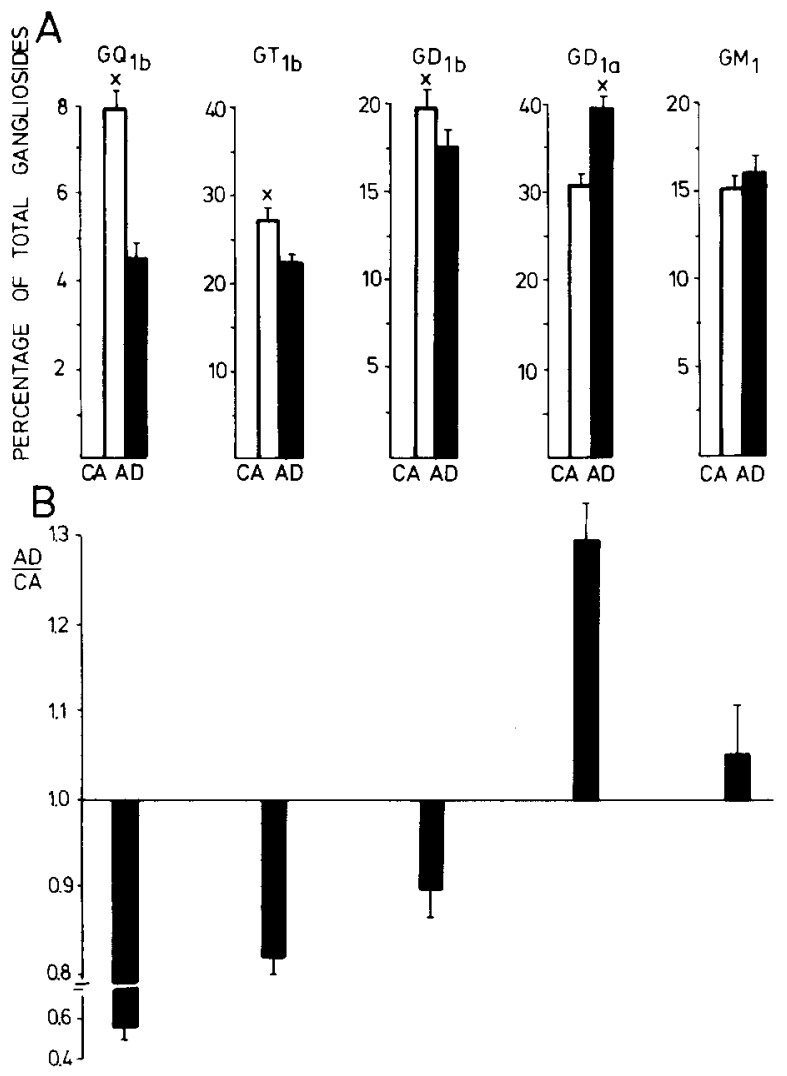

Fig. 2. Distribution of the major gangliosides in the different hippocampal areas of unoperated control rats. A: percentage of individual ganglioside in area dentata, ( $A D$, closed bars) and CA1-CA3 (CA, open bars). B: ratio AD/CA. Data represent the mean values \pm S.E.M. GQ1b, GT1b, GD1b, GD1a, ${ }^{*} P<0.001$ $A D$ vs CA values, GM1 not significant, $n=10$. innervation and extensively denervated the outer twothirds of the dendritic tree of the dentate granule cells.

To define the nature of any changes in ganglioside concentration and relative distribution following lesion, the hippocampus was dissected into two areas: the $\mathrm{AD}$ and the rest of the hippocampus comprising pyramidal cells from CA. This procedure was chosen since $\mathrm{AD}$ is the principal area of entorhinal input into the hippocampus and therefore this area was expected to exhibit the most pronounced changes following lesion. In unoperated animals no conspicuous differences in ganglioside content were noted for the two hippocampal areas studied when right and left hippocampus were compared $(15 \pm 1.4$ in $\mathrm{AD}$ area vs $14 \pm 1.3$ in $\mathrm{CA}$ area nmol $\mathrm{NeuAc/mg}$ of protein). However, examination of the distribution of individual gangliosides in $\mathrm{AD}$ and $\mathrm{CA}$ in unoperated animals provided an unexpected result: interesting regional differences were observed between these areas. In all the cases $\mathrm{AD}$ contained more GD1a
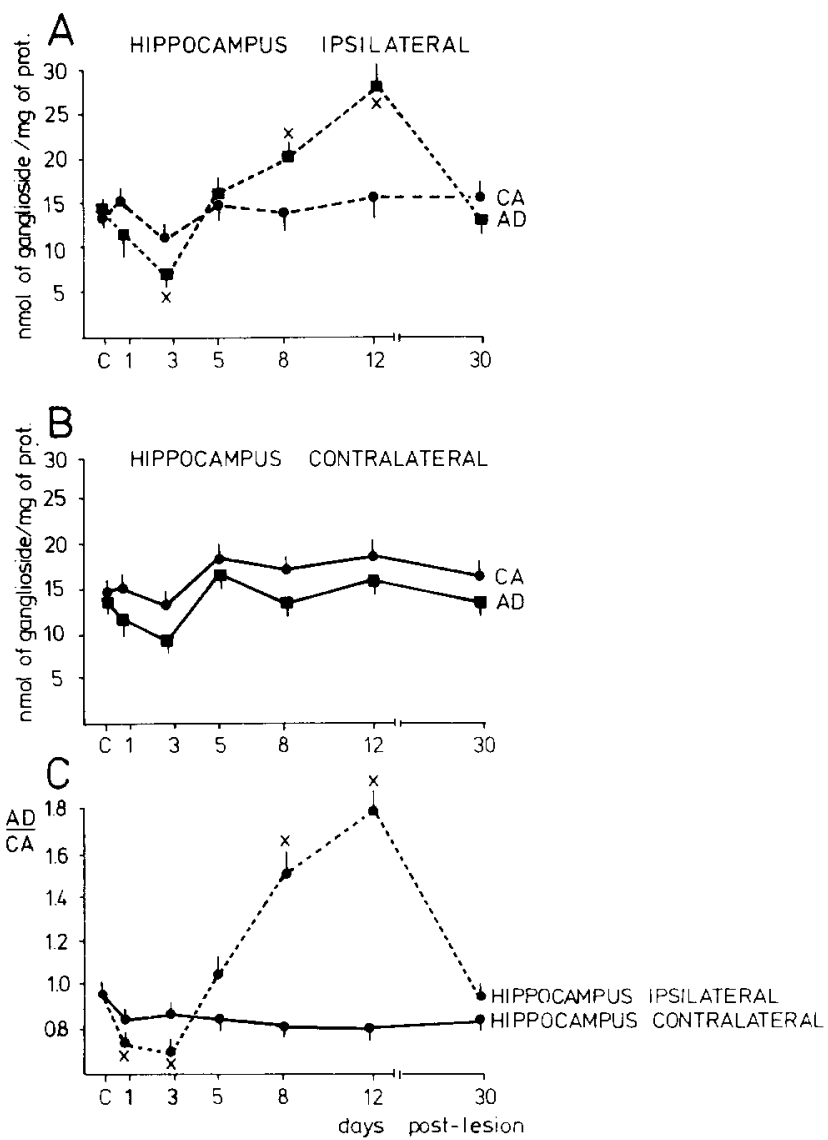

Fig. 3. Changes of total ganglioside content (nmol of sialic acid/mg of protein) at different times after entorhinal lesion. A: area dentata (AD $\square--\square)$ and $\mathrm{CA} 1-\mathrm{CA} 3$ (CA - - - ) ipsilateral to the lesion. $\mathrm{B}: \mathrm{AD}(\mathbf{\square})$ and $\mathrm{CA}(-)$ contralateral to the lesion. $\mathrm{C}$ : ratio of $\mathrm{AD} / \mathrm{CA}$ in the hippocampus ipsilateral (--O) and contralateral $(-)$ ) to the lesion. Values represent the means \pm S.E.M.; 4 lesion animals and 6 controls (C, without lesion). ${ }^{*} P<$ 0.01 
$(28.5 \pm 3 \%)$ with respect to $\mathrm{CA}$. The opposite was observed for GQ1b (48.2 $\pm 3 \%)$, GT1b $(18.5 \pm 2 \%)$ and GD1b $(10.5 \pm 2 \%)$, while the distribution of GM1 was the same in the two areas (Fig. 2A,B).

Fig. 3 illustrates the concentration of gangliosides in the hippocampal areas studied following entorhinal lesion. As can be seen, total ganglioside content decays 3 days postlesion (dpl) in the $\mathrm{AD}$ region ipsilateral to the lesion and after that began to increase reaching a
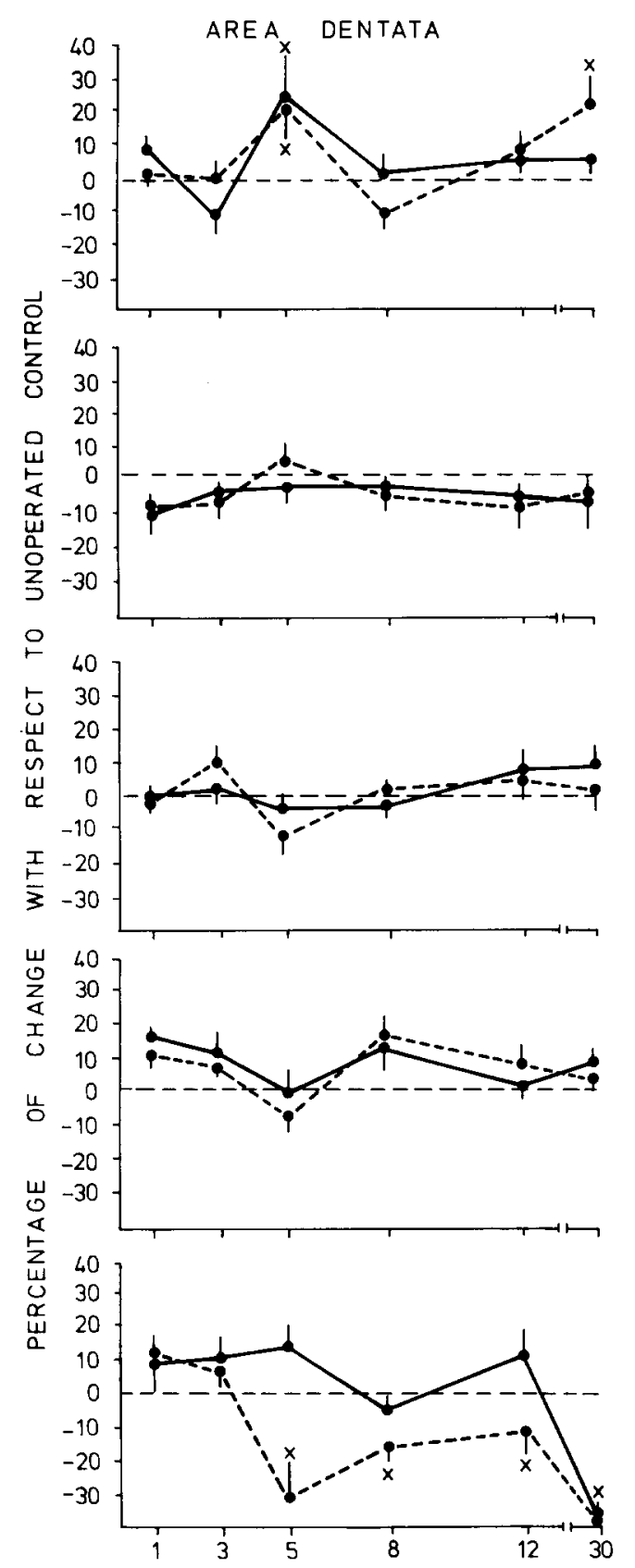

maximum at $12 \mathrm{dpl}$. By $30 \mathrm{dpl}$ the total ganglioside content had returned to control value. The concentration of gangliosides in the CA ipsilateral also showed a decline and a slight increase at $5 \mathrm{dpl}$.

Looking at the contralateral hippocampus (Fig. 3B) changes were not as pronounced as in its ipsilateral counterpart, although at $3 \mathrm{dpl}$ a decrease in $\mathrm{AD}$ region was found and there was a tendency in CA region to remain in values above the unoperated control in later
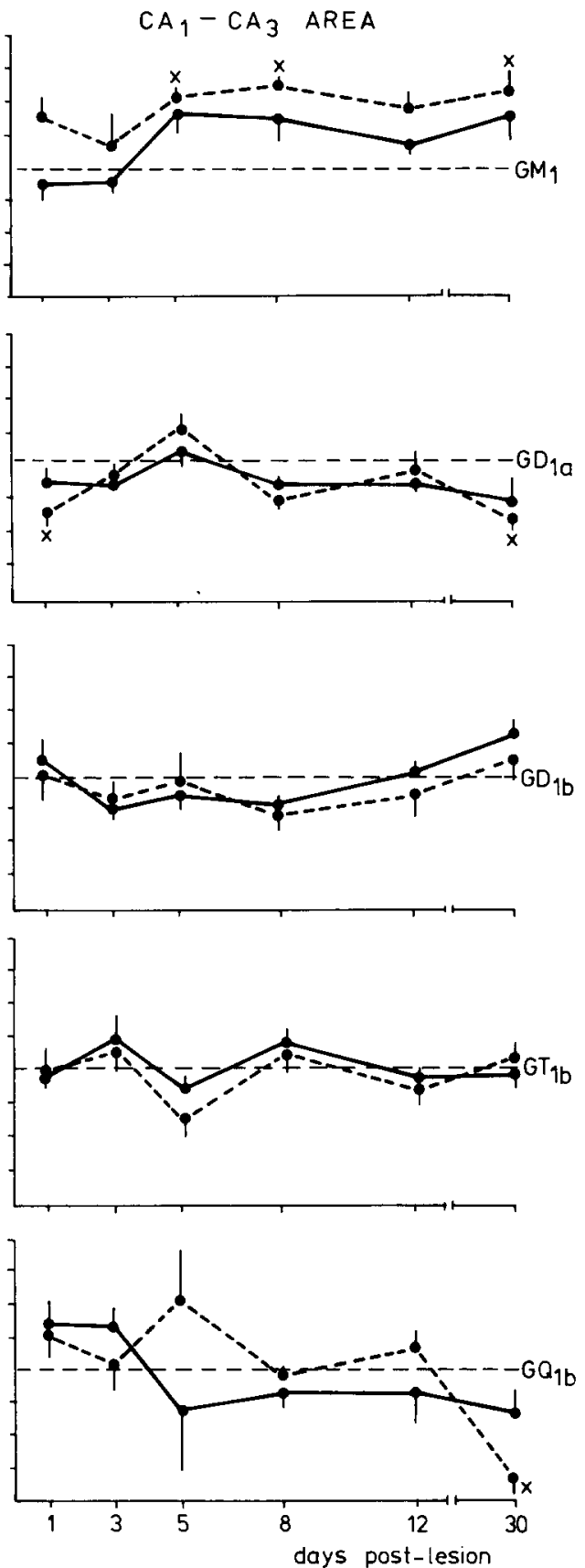

Fig. 4. Percentage of changes of major gangliosides following entorhinal lesion in the AD and CA hippocampal areas with respect to the unoperated control animals, ipsilateral ( as percentage of total ganglioside content in the same area (AD ipsi/contralateral, $\mathrm{CA}$ ipsi/contralateral) with respect to unoperated controls ( $0 \%$ change). Samples of lesions and controls were done on the same HPTLC plate. Values represent the average of 4 lesion animals and 3 unoperated controls per time point. ${ }^{*} P<0.05$. 
time. From all the measurements the ratio AD/CA was calculated for the ipsilateral and contralateral hippocampus. This ratio gives an indication of the preponderance of changes in either the $\mathrm{AD}$ or $\mathrm{CA}$ area. Fig. $3 \mathrm{C}$ shows the decline in the AD/CA ratio at 1 and $3 \mathrm{dpl}$ and the dramatic increase at 8 and $12 \mathrm{dpl}$ in the hippocampus ipsilateral to the lesion. A moderate decline was observed in all the periods studied in the contralateral hippocampus. From both areas of ipsilateral as well as contralateral hippocampus, taken at different times postlesion, the distribution of individual gangliosides was studied. As can be seen in Fig. 4, the most striking changes were found for GM1 and GQ1b. At all the time points studied after lesion, GM1 showed around 20\% increase in CA ipsilateral to the lesion. In AD, GM1 increased $20 \%$ and $25 \%$ at $5 \mathrm{dpl}$ ipsilateral and contralateral, respectively. GD1a showed a decline at 1 and 30 $\mathrm{dpl}$ and a slight increase at $5 \mathrm{dpl}$ in CA ipsilateral to the lesion. A pronounced decline in GQ1b was observed in $\mathrm{AD}$ ipsilateral to the lesion and remained below the control value for all the period studied, also a decline was observed in $\mathrm{AD}$ contralateral to the lesion at $30 \mathrm{dpl}$. In $\mathrm{CA}$ a pronounced decline was found for GQ1b in the ipsilateral hippocampus at $30 \mathrm{dpl}$.

Analysis of gangliosides in lesioned animals after precursor administration of radioactive precursor

In order to obtain more information about ganglioside changes following entorhinal lesion, we studied ganglioside biosynthesis in the deafferented hippocampal regions by intraventricular administration of $\left[{ }^{3} \mathrm{H}\right]$ glucosamine. Unfortunately, a high variation in the total content of radioactive gangliosides of the two areas analyzed was found among different animals, presumably because of differences in the spreading of the injected labelled precursor. Therefore, we were not able to estimate total ganglioside biosynthesis of the two regions separately. However, when the data of the ganglioside biosynthesis from $\mathrm{AD}$ and $\mathrm{CA}$ were pooled, and expressed as the total radioactive ganglioside from hippocampus ipsilateral or contralateral to the lesion, reproducible values were obtained. Furthermore, although there was some variation in the total amount of biosynthetically labelled gangliosides, the relative percentages of individual gangliosides, when the two areas were studied separately, were found to be comparable among different animals.

Fig. 5 shows the dramatic increase in the total ganglioside biosynthesis at 5 and $8 \mathrm{dpl}$ in the hippocampus ipsilateral to the lesion. The changes in the contralateral hippocampus were not as pronounced as those ipsilateral; however, as can be seen in Fig. 5, the incorporation of radioactivity was always $3-5$ times less in contralateral as compared to ipsilateral hippocampus,

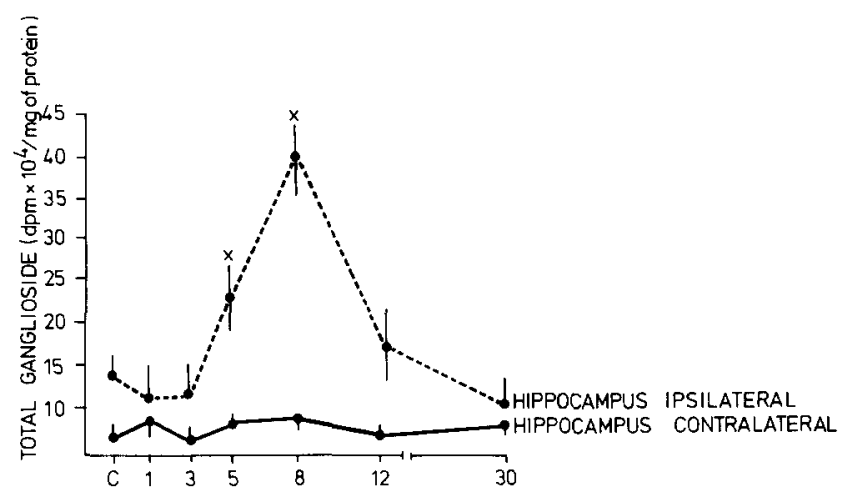

Fig. 5. Total radioactive ganglioside content, after intraventricular administration of $\left[{ }^{3} \mathrm{H}\right]$ glucosamine, following entorhinal lesion. Hippocampus ipsilateral (---) and contralateral (-) to the lesion. Data represent mean value \pm S.E.M. of 3 lesion animals at each time point and 4 controls $(\mathrm{C}$, without lesion). $P<0.05$.

also in the unoperated animals. This indicates that the diffusion of the radioactive precursor was not the same for both hippocampi, when the precursor was injected in one of the brain ventricles.

Analysis of the distribution of individual radioactive gangliosides is illustrated in Fig. 6. As can be seen the most important changes were observed in GM1 ipsilateral and contralateral to the lesion in both, $\mathrm{AD}$ and $\mathrm{CA}$. Biosynthesis of GM1 increased $60 \%$ at $3 \mathrm{dpl}$ in $\mathrm{AD}$ ipsilateral to the lesion. A pronounced increase was also seen at $5,8,12$ and $30 \mathrm{dpl}$ in $\mathrm{AD}$ contralateral. A pronounced decrease in CA contralateral was observed at $1 \mathrm{dpl}$ and a significant increase at $30 \mathrm{dpl}$ in $\mathrm{CA}$ area contralateral and ipsilateral. A decline of GD1a at $1 \mathrm{dpl}$ followed by an increase at $3 \mathrm{dpl}$ was observed in CA. The same increase was observed for GT1b in CA at 1 and 3 dpl. At 5 and $8 \mathrm{dpl}$ a decrease for GD1b in AD was found.

In these experiments we could not reliably measure a possible change in GQ1b due to the proximity of its HPTLC band to GT1b, which is 5-8 times more concentrated. Thus it was impossible to avoid some contamination of the scraped-off GQ1b band by GT1b, making accurate quantification difficult.

As a control, changes in gangliosides in the cerebellum are shown in Fig. 6 for comparison. Cerebellum, being a region of the brain that does not participate in sprouting after entorhinal lesion, did not show any changes in the ganglioside pattern.

\section{DISCUSSION}

The present observation indicates that postlesion afferent reorganization of the hippocampus of adult rats leads to important modifications in endogenous ganglioside content and distribution. 

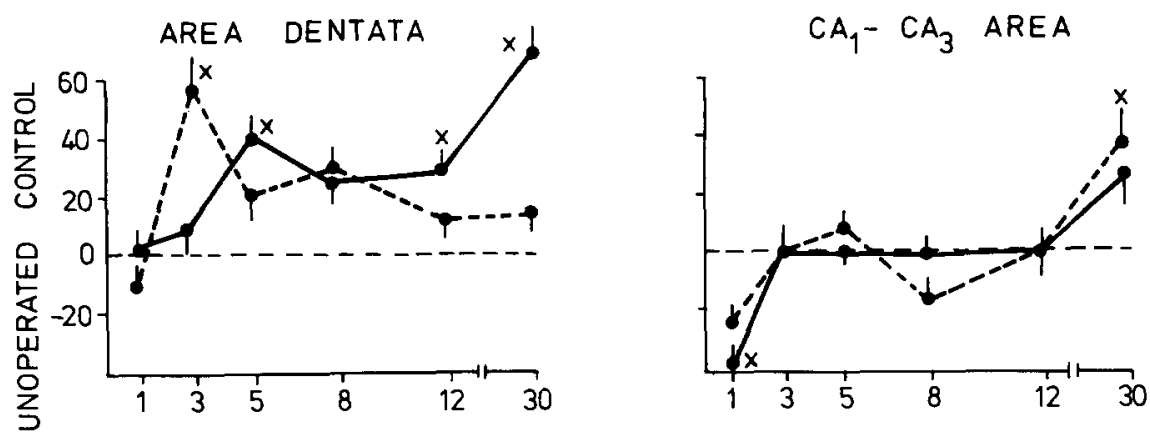

CERE BELLUM
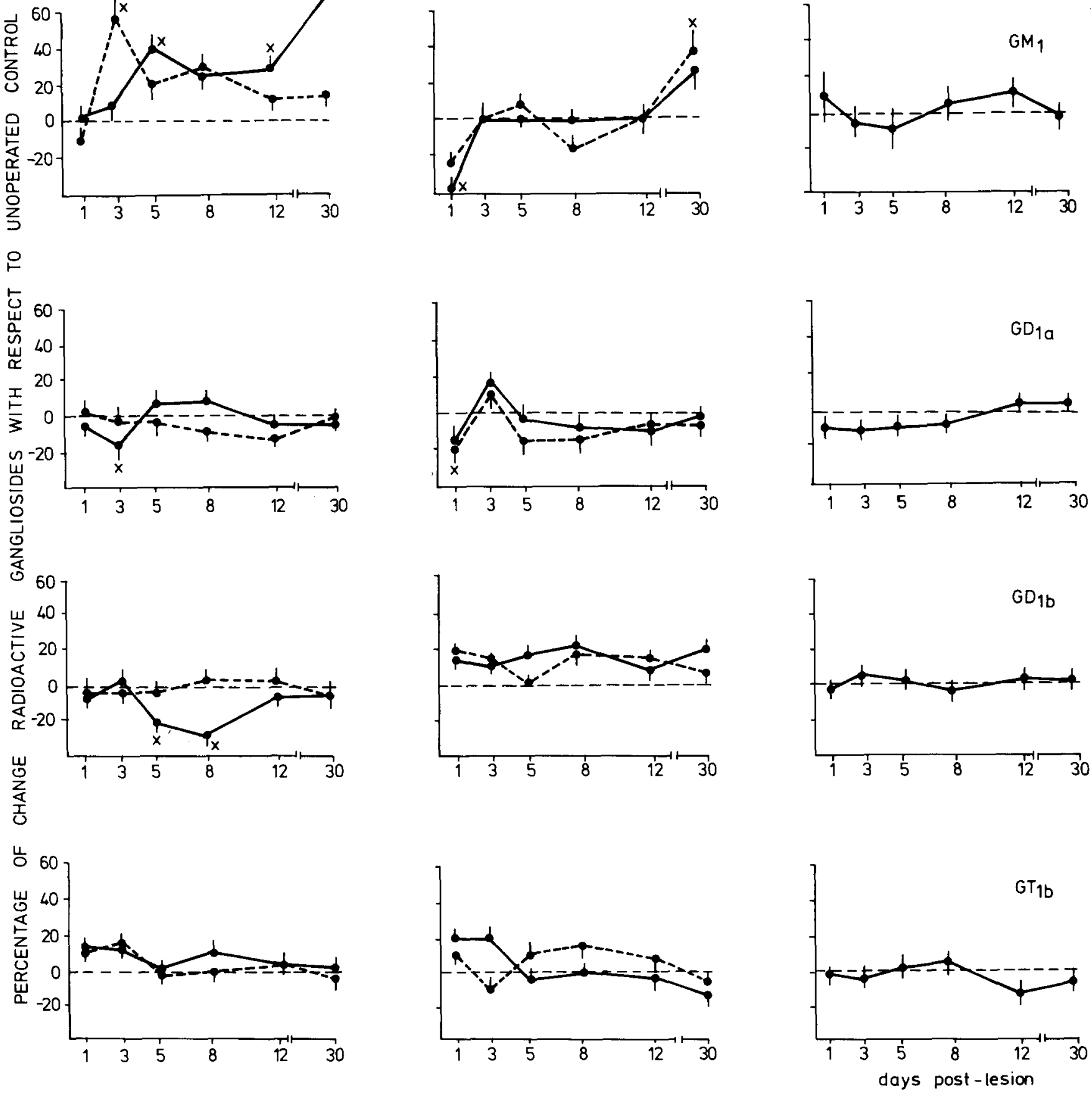

Fig. 6. Percentage of changes in radioactive gangliosides, with respect to unoperated control animals, following entorhinal lesion. Ipsilateral (-) and contralateral (-) area to the lesion. For comparison the percentage of changes in gangliosides from cerebellum are shown in the same figure. Data represent mean values \pm S.E.M. of 3 different lesion animals at each time point and 3 controls. $P<0.05$.

The analysis of the time course of the increased content of gangliosides shows that this change parallels the time course ${ }^{5}$ of axonal proliferation and sprouting.

Prior to the evaluation of these results it is interesting to analyze in unoperated control animals the different profiles of ganglioside distribution of the two hippocampal areas studied. In all these cases a higher percentage of GD1a in AD as compared to $\mathrm{CA}$ was found, whereas GQ1b, GT1b and GD1b were higher in CA.

This differential distribution of ganglioside may reflect the cellular composition of the two areas studied, pyramidal neurons as compared to the granular neurons of the AD. Alternatively it may reflect differences in the number of synapses, type of neuronal membrane, or kind of neurotransmitter used by the neurons of these areas. For example, there is a minor ganglioside, Chol 1, which 
seems to be specific for cholinergic terminals (unpublished observation). Similar differences between hippocampal regions were found both in adult rat and during development ${ }^{16}$. Evidence for some association between specific gangliosides and discrete neuronal cell types in the mouse cerebellum has also been reported ${ }^{52}$.

In lesioned animals, there was a temporal correlation between the increase in the biosynthesis of gangliosides as studied by radioactive precursor injection and the total 'cold' ganglioside content (Figs. 3-6). The drop observed in the earlier days postlesion may reflect the loss of synapses ${ }^{14}$, not only in the AD ipsilateral to the lesion but also in the contralateral region where nearly $20 \%$ of the synapses are lost after entorhinal lesion ${ }^{15}$. When we analyzed the individual ganglioside profile we found that the biosynthesis of GM1 preceded the observed increase in total GM1. However, this correlation was not found for the other gangliosides. Perhaps the most likely interpretation is that one particular ganglioside is made in one region and transported to the other ${ }^{18}$. Also, other structures which are participating in the remodeling process, e.g. the septum, could send gangliosides to the terminals localized in the lesioned area and thus contribute to the changes observed. The observed increase in GM1 biosynthesis on the contralateral side at $30 \mathrm{dpl}$ is difficult to understand, but might reflect a compensatory reaction of the contralateral side, as was reported by Hoff et al. ${ }^{15}$

In studies of cellular correlates of postlesion growth, 3 potential cellular participants must be considered: (1) the sprouting presynaptic neuron, (2) the postsynaptic neuron being reinnervated, and (3) the glial cells. Though glia cell proliferation and migration occurs early after lesion $(1-4 \mathrm{dpl})^{13,43}$, this process appears to be waning by the time of the onset of neuronal growth and the observed increase in the ganglioside content at $8-12 \mathrm{dpl}$. Even more, at the time of maximum glial proliferation, i.e. 1-4 dpl, a decline in the content of ganglioside was found (Fig. 3).

Another candidate for the ganglioside changes observed are the astrocyte cells. Recently Gage et al. ${ }^{12}$ reported that following perforant path lesion, which produces similar denervation to electrocoagulation of the entorhinal cortex, resulted in an increase in glial fibrillary acidic protein immunoreactivity in the outer molecular layer of the dentate gyrus. This increase is produced at 8 days postlesion and remained until day 30 . However, if we consider the total gyrus dentate area, as in our case, no changes in the number of astrocytes can be observed $^{12}$. Thus it is reasonable to assume that the changes in ganglioside content reported in this study cannot be attributed to the increased number of astrocyte cells. On the other hand, ganglioside content and distribution in astrocyte cells is a controversial problem. Recently it has been reported ${ }^{2}$ that astrocytes had a similar ganglioside pattern and a considerable ganglioside content $(2.79 \mu \mathrm{g}$ $\mathrm{NeuAc/mg}$ protein) to neurons isolated from the same source. However in this report it was also found that purified astrocytes had no $\beta$-Ga1NacT-1, a key transferase enzyme in the synthesis of ganglio-series gangliosides. This may indicate that gangliosides in astrocyte cells can be derived from a contamination of isolated astrocytes by neuronal processes during the fraction preparation or, as the authors propose, astrocyte gangliosides may be transferred from neurons to astrocytes. Also astrocytes in cell culture exhibit mainly GM3 ganglioside and not the others, and no changes in ganglioside pattern were observed when comparing flat with stellate astrocytes, the latter resembling astrocytes in reactive gliosis ${ }^{29}$.

Although these reasons do not totally eliminate glial cells as candidates for ganglioside changes, the above considerations seem to indicate that they are not responsible for the changes found. Despite the fact that we analyzed the ganglioside content and distribution in the very 'crude' dissected hippocampus and not in a particular axonal system, we think that the changes observed were primarily related to events in sprouting neurons and/or axons, since our results exhibit an excellent temporal correlation between ganglioside changes and proliferation of new axonal fibers as reported in the literature $\mathrm{e}^{5,15,26,33,49,55}$

Similarly, there is a good correlation with the known increase in neurotrophic factors in the hippocampal region after the same type of lesion ${ }^{34,35}$. Gangliosides have been postulated to be related with neurotrophic activities $^{10,22,23,31,50}$. However, the observation that gangliosides do not possess intrinsic trophic activity, but can enhance the capacity of neurons to respond to other neurotrophic activities ${ }^{23}$, suggests that their most probable participation in regeneration phenomena is perhaps by facilitating or modulating cellular responses to neurotrophic substances. However, the mechanisms underlying this role of gangliosides is currently obscure.

In any case, the period between 8 and $12 \mathrm{dpl}$, which represents a time of active lesion-induced synaptogenesis in the surviving afferent system (see refs. above), shows the most dramatic increase in ganglioside content observed in this study. These results are consistent with in vivo labelling studies showing that axonal transport of radiolabelled gangliosides undergoes a dramatic increase during regeneration of goldfish optic axon ${ }^{48}$.

It is tempting to suggest that synthesis of certain membrane components is selectively enhanced in order to provide the optimum conditions for proliferating afferent fibers, and/or for lesioned postsynaptic neurons to accept the new synaptic connections. 


\section{REFERENCES}

1 Agnati, L., Fuxe, K., Calza, L., Benfenati, F., Cacicchioli, L., Toffano, G. and Golstein, M., Gangliosides increase the survival of lesioned nigral dopamine neurons and favour the recovery of dopamine synaptic function in striatum of rats by collateral sprouting, Acta Physiol. Scand., 119 (1983) 347-363.

2 Byrne, M., Farooq, M., Sbasching-Adler, M., Norton, W. and Ledeen, R., Ganglioside content of astroglia and neurons isolated from maturing rat brain: consideration of the source of astroglial gangliosides, Brain Research, 461 (1988) 87-97.

3 Byrne, M., Ledeen, R.W., Roisen, F.J., Yorke, G. and Sclafani, J.R., Gangliosides induced neuritogenesis: verification that the gangliosides are the active agents, and comparison of molecular especies, J. Neurochem., 41 (1983) 1214-1222.

4 Casamenti, F., Bracco, L., Bartolini, L. and Pepeu, G., Effects of ganglioside treatment in rats with a lesion of the cholinergic forebrain nuclei, Brain Research, 338 (1985) 45-52.

5 Cotman, C.W. and Nadler, V.J., Reactive synaptogenesis in the hippocampus. In C.W. Cotman (Ed.), Neuronal Plasticity, Raven, New York, 1978, pp. 227-271.

6 Dahms, N.M. and Schaar, R.L., Ganglioside composition is regulated during differentiation in the neuroblastoma and glioma hybrid cell line NG 108-115, J. Neurochem., 3 (1983) 806-817.

7 Dimpfel, W., Möller, W. and Mengs, U., Ganglioside-induced neurite formation in cultured neuroblastoma cells. In $M$. Rapport and A. Gorio (Eds.), Gangliosides in Neurological and Neuromuscular Function, Development and Repair, Raven, New York, 1981, pp. 119-134.

8 Dreyfus, H., Louis, J., Harth, S. and Mandel, P., Gangliosides in cultured neurons, Neuroscience, 5 (1980) 1647-1655.

9 Dreyfus, H., Hart, S., Massarelli, R. and Louis, J., Mechanisms of differentiation in cultured neurons. Involvement of gangliosides. In M. Rapport and A. Gorio (Eds.), Gangliosides in Neurological and Neuromuscular Function, Development and Repair, Raven, New York, 1981, pp. 151-170.

10 Facci, L., Leon, A., Toffano, G., Sonnino, S., Ghidoni, R. and Tettamanti, G., Promotion of neuritogenesis in mouse neuroblastoma cells by exogenous gangliosides. Relationship between the effect and the cell association of ganglioside GM1, $J$. Neurochem., 42 (1984) 299-305.

11 Fass, B. and Ramirez, J., Effect of ganglioside treatments on lesion-induced behavioral impairments and sprouting in the CNS, J. Neurosci. Res., 12 (1984) 445-458.

12 Gage, F., Olejniczak, P. and Armstrong, D., Astrocytes are important for sprouting in the septohippocampal circuit, Exp Neurol., 102 (1988) 2-13.

13 Gall, C., Rose, G. and Lynch, G., Proliferative and migratory activity of glial cells in the partially deafferented hippocampus, J. Comp. Neurol., 183 (1979) 539-550.

14 Hess, H.H., Bass, N.H., Thalheimer, C. and Devarakonda, R., Gangliosides and the architecture of human and rat somatosensory isocortex, J. Neurochem., 26 (1976) 1115-1121.

15 Hoff, S.F., Scheeff, S.W., Kwan, A.Y. and Cotman, C.W., A new type of lesion-induced synaptogenesis: I. Synaptic turnover in non-denervated zones of the dentate gyrus in young adult rats, Brain Research, 222 (1981) 1-13.

16 Irwin, L.N. and Irwin, C.C., Developmental changes and regional variation in the ganglioside composition of the rat hippocampus, Dev. Brain Res., 4 (1982) 481-485.

17 Karpiak, S.E., Ganglioside treatment improves recovery of alternation behavior following unilateral entorhinal cortex lesion, Exp. Neurol., 81 (1983) 330-339.

18 Landa, C., Maccioni, H. and Caputto, R., The site of synthesis of gangliosides in the chick optic system, $J$. Neurochem., 33 (1979) 825-838.

19 Landa, C.A. and Moscona, A.A., Changes in gangliosides profile in chick embryo retina: studies on tissues and cell culture, Int. J. Dev. Neurosci., 3 (1985) 77-88.

20 Landa, C.A. and Moscona, A.A., Gangliosides in postmitotic retina of chick embryo. Changes in vivo and in cell culture, Dev. Brain Res., 21 (1985) 193-202.

21 Ledeen, R.W., Gangliosides. In A. Lajtha (Ed.), Handbook of Neurochemistry, Vol. 3, Plenum, New York, 1983, pp. 41-90.

22 Leon, A., Facci, L., Benvegnu, D. and Toffano, G., Morphological and biochemical effects of gangliosides in neuroblastoma cells, Dev. Neurosci., 5 (1982) 108-114.

23 Leon, A., Dal Toso, R., Presti, D., Benvegnu, D., Facci, L., Kirschner, G., Tettamanti, G. and Toffano, G., Development and survival of neurons in dissociated fetal mesencephalic serum-free cell cultures: II. Modulatory effects of gangliosides, J. Neurosci., 8 (1988) 746-753.

24 Loesche, J. and Steward, O., Behavioral correlates of denervation and reinnervation of the hippocampal formation of the rat: recovery of alternation performance following unilateral entorhinal cortex lesion, Brain Res. Bull., 2 (1977) 31-39.

25 Lowry, O., Rosebrough, N., Farr, A. and Randall, R., Protein measurement with the Folin phenol reagent, J. Biol. Chem., 193 (1951) 265-275.

26 Lynch, G., Gall, C. and Cotman, C., Temporal parameters of 'axonal sprouting' in the brain of adult rat, Exp. Neurol., 54 (1977) 179-183.

27 Margolis, R.K., Salton, S.R. and Margolis, R.U., Complex carbohydrates of cultures PC12 pheochromocytoma cells. Effects of nerve growth factor and comparison with neonatal and mature brain, J. Biol. Chem., 258 (1983) 4110-4117.

28 Masco, D. and Seifert, W., Incorporation of ganglioside GM1 into rat brain after intraventricular administration, Neurosci. Res. Commun., (1988) 141-149.

29 Masco, D., Flott, B. and Seifert, W., Astrocytes in cell culture incorporate GM1 ganglioside, Glia, in press.

30 Merat, A. and Dickerson, J.W., The effect of development on the gangliosides of rat and pig brain, J. Neurochem., 20 (1973) 873-880.

31 Morgan, J.I. and Seifert, W., Growth factors and gangliosides: a possible perspective in neuronal growth control, Supramol. Struct., 10 (1979) 111-124.

32 Nadler, J., Cotman, C. and Lynch, G., Biochemical plasticity of short-axon interneurons: increased glutamate decarboxylase activity in the denervated area of rat dentate gyrus following entorhinal cortex lesion, Exp. Neurol., 45 (1974) 403-413.

33 Nadler, J., Cotman, C., Paoletti, C. and Lynch, G., Histochemical evidence of altered development of cholinergic fibers in the rat dentate gyrus following lesion. I. Time course after complete unilateral lesion at various ages, J. Comp. Neurol., 171 (1977) 561-588.

34 Nieto-Sampedro, M., Lewis, E., Cotman, C., Manthorpe, M., Skaper, S., Barbin, G., Longo, F. and Varon, S., Brain injury causes a time-dependent increase in neurotrophic activity at the lesion site, Science, 217 (1982) 860-861.

35 Nieto-Sampedro, M. and Cotman, C., Growth factor induction and temporal order in central nervous system repair. In C.W. Cotman (Ed.), Synaptic Plasticity, Guilford, New York, 1985, pp. 407-455.

36 Oderfeld-Nowak, B., Skup, M., Ulas, J., Jezierska, M., Gradkowska, M. and Zaremba, M., Effect of GM1 ganglioside treatment on postlesion responses of cholinergic enzymes in rat hippocampus after various partial deafferentations, $J$. Neurosci. Res., 12 (1984) 409-420.

37 Panzeta, P., Gravotta, D. and Maccioni, H., Biosynthesis and expression of gangliosides during differentiation of chick embryo retina cells in vitro, $J$. Neurochem., 49 (1987) 1763-1771.

38 Petrosini, L., Behavioural recovery from unilateral vestibular lesion is facilitated by GM1 ganglioside treatment, Behav. Brain Res., 23 (1987) 117-126.

39 Purpura, D.P. and Baker, H.J., Neurite induction in mature cortical neurons in feline GM1 ganglioside storage disease, Nature (Lond.), 226 (1977) 553-554.

40 Ramirez, J., Fass, B., Karpiak, S. and Steward, O., Ganglioside treatments reduce hyperactivity after bilateral lesions of the 
entorhinal cortex, Neursoci. Lett., 75 (1987) 283-287.

41 Ramirez, J., Fass, B., Kilfoil, T., Henschel, B., Grones, W. and Karpiak, S., Ganglioside-induced enhancement of behavioral recovery after bilateral lesion of the entorhinal cortex, Brain Research, 414 (1987) 85-90.

42 Roisen, F., Bartfeld, H., Nagele, R. and Yorke, G., Ganglioside stimulation of axonal sprouting in vitro, Science, 214 (1981) $577-578$

43 Rose, G., Lynch, G. and Cotman, C., Hypertrophy at redistribution of astrocytes in the deafferented dentate gyrus, Brain Res. Bull., 1 (1976) 87-92.

44 Rösner, H., Gangliosides changes in the chicken optic lobes biochemical indicators of brain development and maturation, Brain Research, 236 (1982) 49-61.

45 Rösner, H. and Rahmann, H., Ontogeny of vertebrate brain gangliosides. In H. Rahmann (Ed.), Gangliosides and Modulation of Neuronal Functions, NATO ASI Series, Springer, Berlin, 1987, pp. 373-390.

46 Sabel, B.A., Slavin, M.D. and Stein, D.G., GM1 ganglioside treatment facilitates behavioral recovery from bilateral brain damage, Science, 225 (1984) 340-342.

47 Sabel, B.A., Dunbar, G.L., Butler, W.M. and Stein, D.J., GM1 ganglioside stimulates neuronal reorganization and reduces rotational asymmetry after hemitransections of the nigrostriatal pathway, Exp. Brain Res., 60 (1985) 27-37.

48 Sbaschning-Agler, M., Ledeen, R.W., Grafstein, B. and Alpert, R.M., Gangliosides changes in the regenerating goldfish optic system: comparison with glycoproteins and phospholipids, $J$. Neurosci., 12 (1984) 221-232.

49 Scheff, S.W., Benardo, L.S. and Cotman, C.W., Decline in reactive fiber growth in the dentate gyrus of aged rats compared to young adult rats following entorhinal cortex removal, Brain Research, 199 (1980) 21-38.

50 Schwartz, M. and Spirman, N., Sprouting from chick embryo dorsal root ganglia induced by nerve growth factor is specifically inhibited by affinity purified anti-GM1 antibody, Proc. Natl. Acad. Sci. U.S.A., 79 (1982) 6080-6083.

51 Seifert, W., Gangliosides in nerve cell cultures. In M. Rapport and A. Gorio (Eds.), Raven, New York, 1981, pp. 99-117.

52 Seyfried, T.N., Yu, R.K. and Miyazawa, N., Differential cellular enrichment of gangliosides in the mouse cerebellum: analysis using neurological mutants, J. Neurochem., 38 (1982) 551-559.

53 Seyfried, T.N., Miyazawa, N. and Yu, R.K., Cellular localization of gangliosides in the developing mouse cerebellum: analysis using the weaver mutant, J. Neurochem., 41 (1983) 491-505.

54 Seyfried, T.N., Bernard, D.J. and Yu, R.K., Cellular distribution of gangliosides in the developing mouse cerebellum: analysis using the staggerer mutant, J. Neurochem., 431 (1984) 11521162.

55 Steward, O. and Loesche, J., Quantitative autoradiographic analysis of the time course of proliferation of the entorhinal afferents in the dentate gyrus denervated by ipsilateral entorhinal lesions, Brain Research, 125 (1977) 11-21.

56 Suzuki, K., The pattern of mammalian brain gangliosides. III. Regional and developmental differences, J. Neurochem., 12 (1965) 969-979.

57 Svennerholm, L., Quantitative estimation of the sialic acids. II. A colorimetric resorcinol hydrochloric acid method, Biochim. Biophys. Acta, 24 (1957) 604-611.

58 Svennerholm, L., Chromatographic separation of human brain gangliosides, J. Neurochem., 10 (1963) 613-623.

59 Svennerholm, L. and Fredman, P., A procedure for the quantitative isolation of brain gangliosides, Biochim. Biophys. Acta, 617 (1980) 97-109.

60 Wiegant, H., The gangliosides, Adv. Neurochem., 4 (1982) 149-223.

61 Willinger, M., The expression of GM1 ganglioside during neuronal differentiation. In M. Rapport and A. Gorio (Eds.), Gangliosides in Neurological and Neuromuscular Function, Development and Repair, Raven, New York, 1981, pp. 19-27.

62 Yavin, E. and Yavin, Z., Ganglioside profile during neural tissue development. Acquisition in the prenatal rat brain and cerebral cell culture, Dev. Neurosci., 2 (1979) 25-37.

$63 \mathrm{Yu}$, R., Macala, L., Taki, T., Weinfeld, H. and Yu, F, Developmental changes in ganglioside composition and synthesis in embryonic rat brain, J. Neurochem., 50 (1988) 1825-1829. 